\title{
HOMOGENIZATION of SYSTEMS of PARTIAL DIFFERENTIAL EQUATIONS
}

\author{
A. Bensoussan \\ University Paris Dauphine
}

November 9, 2003

\section{INTRODUCTION}

In this paper, we consider the class of systems of nonlinear partial differential equations, which has been lengthily studied by Prof J. FREHSE and the A., with application to stochastic differential games with $N$ players. In particular, we refer to the book, A.BENSOUSSAN, J. FREHSE [1]. The regularity theory is instrumental to prove the existence of equilibriums in noncooperative games. The objective in this paper is to show that regularity theory is also extremely useful for obtaining the limit of problems with small parameters, like in homogenization. The methods used for scalar equations cannot extend, and the regularity results become instrumental

\section{STATEMENT of THE PROBLEM and RESULTS}

\subsection{NOTATION}

We consider a family of matrices $a^{\epsilon}(x)$ satisfying

$$
a^{\epsilon}(x) \text { is measurable on } R^{n}
$$

$$
\begin{gathered}
a^{\epsilon}(x) \xi . \xi \geq \alpha|\xi|^{2}, \forall \xi \in R^{n}, \alpha>0 \\
\left(a^{\epsilon}\right)^{-1}(x) \xi . \xi \geq \alpha_{0}|\xi|^{2}, \forall \xi \in R^{n}, \alpha_{0}>0 .
\end{gathered}
$$

We shall say that $a^{\epsilon}$ belongs to the class $M\left(\alpha, \alpha_{0}\right)$. Let $\Omega$ be a smooth bounded domain of $R^{n}$. Following the theory of abstract homogenization introduced by L. TARTAR [6] and F. MURAT,L. TARTAR [5], we shall consider the following properties. There exists a sequence of vectors $v^{\epsilon}(x) \in R^{n}$, such that

$$
\begin{gathered}
v^{\epsilon} \in\left(H^{1}(\Omega)\right)^{n} \text { and } v^{\epsilon} \rightarrow x \text { in }\left(H^{1}(\Omega)\right)^{n} \text { weakly } \\
a^{\epsilon}\left(D v^{\epsilon}\right)^{*} \rightarrow a \text { in }\left(L^{2}(\Omega)\right)^{n \times n} \text { weakly } \\
\operatorname{div}\left(a^{\epsilon}\left(D v^{\epsilon}\right)^{*}\right) \rightarrow \operatorname{div} a \text { in }\left(H^{-1}(\Omega)\right)^{n} \text { strongly }
\end{gathered}
$$

Note that

$$
\left(D v^{\epsilon}\right)_{i j}=\frac{\partial v_{i}^{\epsilon}}{\partial x_{j}} .
$$

It is a classical result that $a$ belongs to $M\left(\alpha, \alpha_{0}\right)$.

We also assume, for technical reasons,

$$
\left\|D v^{\epsilon}(x)\right\| \leq B, x \in \Omega
$$


We next consider $N$ Hamiltonians $H^{\epsilon, \nu}(x, s, \xi)$, where $s \in R^{N}, \xi \in R^{N \times n}, \nu=1, \cdots, N$, such that

$$
\begin{aligned}
& \left.H^{\epsilon, \nu}(x, s, \xi)\right|_{\left\{\xi^{\nu}=0\right\}}-H^{\epsilon, \nu}(x, s, \xi)\left\{\xi^{\nu}=0, s^{\nu}=0\right\} \geq \beta^{\nu} s^{\nu}, \text { if } s^{\nu}>0 \\
& \left.H^{\epsilon, \nu}(x, s, \xi)\right|_{\left\{\xi^{\nu}=0\right\}}-H^{\epsilon, \nu}(x, s, \xi)\left\{\xi^{\nu}=0, s^{\nu}=0\right\} \leq \beta^{\nu} s^{\nu}, \text { if } s^{\nu}<0
\end{aligned}
$$

Writing $H^{\epsilon}$ for the vector $H^{\epsilon, \nu}$, we also assume

$$
\left|H^{\epsilon}(x, s, \xi)-H^{\epsilon}\left(x, s^{\prime}, \xi\right)\right| \leq \varpi\left(\left|s-s^{\prime}\right|\right)\left(1+|\xi|^{2}\right)
$$

and

$$
\left|H^{\epsilon}(x, s, \xi)-H^{\epsilon}\left(x, s, \xi^{\prime}\right)\right| \leq \gamma\left|\xi-\xi^{\prime}\right|\left(1+|\xi|+\left|\xi^{\prime}\right|+|s|^{\frac{1}{2}}\right)
$$

where $\varpi: R^{+} \rightarrow R^{+}$is continuous, increasing, $\varpi(0)=0$. Next we assume a special growth assumption on the Hamiltonians

$$
\begin{aligned}
& \left|H^{\epsilon, \nu}(x, s, \xi)\right| \leq K^{\nu}|\xi|\left|\xi^{\nu}\right|+\sum_{\{\mu=1\}}^{\nu} K_{\mu}^{\nu}\left|\xi^{\mu}\right|^{2}+k^{\nu}(x), \nu=1, \cdots, N-1 \\
& \left|H^{\epsilon, N}(x, s, \xi)\right| \leq K^{N}|\xi|^{2}+k^{N}(x)
\end{aligned}
$$

where

$$
K^{\nu}, K_{\mu}^{\nu} \text { are positive constants }, k^{\nu} \geq 0 \in L^{q}(\Omega), q>\frac{n}{2} .
$$

We consider the system of elliptic equations

$$
-\operatorname{div}\left(a^{\epsilon}(x) D u^{\epsilon, \nu}\right)+H^{\epsilon, \nu}\left(x, u^{\epsilon}, D u^{\epsilon}\right)=0, x \in \Omega,\left.u^{\epsilon, \nu}\right|_{\partial \Omega}=0
$$

where $u^{\epsilon}$ denotes the vector of components $u^{\epsilon, \nu}$. The functions $u^{\epsilon, \nu}$ belong to

$$
u^{\epsilon, \nu} \in H_{0}^{1}(\Omega) \times L^{\infty}(\Omega)
$$

In our following development estimates will be proven, which will be uniform in $\epsilon$, so we shall assume the existence of $u^{\epsilon}$ so that (2. 15), (2. 16) hold. We can refer to A.BENSOUSSAN, J. FREHSE [2].

Remark 2.1 There is an additional degree of freedom, related to the ordering of equations in writing the system. Let $\Gamma$ be an $N \times N$ matrix, which is invertible. To $\Gamma$ we associate the transform of $H^{\epsilon}$ denoted $H_{\Gamma}^{\epsilon}$, defined as follows

$$
H_{\Gamma}^{\epsilon}(x, s, \xi)=\Gamma H^{\epsilon}\left(x, \Gamma^{-1} s, \Gamma^{-1} \xi\right) .
$$

Setting

$$
z^{\epsilon}=\Gamma u^{\epsilon}
$$

then $z^{\epsilon}$ is the solution of (2. 15),(2. 16), with $H^{\epsilon}$ replaced by $H_{\Gamma}^{\epsilon}$. We shall need that (2. 8),(2. 9),(2. 10) hold for some transform $H_{\Gamma}^{\epsilon}$, with $\Gamma$ satisfying the Maximum Principle, which means

$$
\Gamma s \geq 0 \Rightarrow s \geq 0
$$

and that (2. 11), (2. 12), (2. 13) hold for another transfom $H_{\Gamma}^{\epsilon}$, not necessarily the same, in particular with $\Gamma$ not satisfying the Maximum Principle. We shall neeed (2. 8),(2. 9),(2. 10) to prove that $z^{\epsilon}$ is bounded. Since $\Gamma$ satisfies the Maximum Principle and is invertible, this implies that $u^{\epsilon}$ is bounded. This being achieved, another transformation, not necessarily satisfying the Maximum Principle preserves the $L^{\infty}$ bound. It permits to show $C^{\delta}$ estimates, which are also valid for $u^{\epsilon}$. In the statement of results, this flexibility will be implicit. 


\section{$2.2 \quad$ STATEMENT of RESULTS}

Our objective is to prove the following

Theorem 2.1 We make the assumptions (2. 1),(2. 2),(2. 3),(2. 4),(2. 5), (2. 6),(2. 7),(2. 8),(2. 9),(2. 10), (2. 11),(2. 12), (2. 13),(2. 14). For the assumptions (2. 8) to (2. 14), we take into account Remark 2.1. Let $u^{\epsilon}$ be a solution of the system (2. 15), then

$$
\left\|u^{\epsilon, \nu}\right\|_{L^{\infty}(\Omega)} \leq C,\left\|u^{\epsilon, \nu}\right\|_{H_{0}^{1}(\Omega)} \leq C
$$

If we pick a subsequence, still denoted $u^{\epsilon, \nu}$, such that then

$$
D u^{\epsilon, \nu}-\left(D v^{\epsilon}\right)^{*} D u^{\nu} \rightarrow 0 \text { in } L^{2}(\Omega) .
$$

Moreover there exist Hamiltonians $H^{\nu}(x, s, \xi)$ satisfying assumptions (2. 8) to (2. 14), with possibly different constants, such that $u$ the vector of components $u^{\nu}$ satisfies the equations

$$
-\operatorname{div}\left(a(x) D u^{\nu}\right)+H^{\nu}(x, u, D u)=0, x \in \Omega,\left.u^{\nu}\right|_{\partial \Omega}=0
$$

\section{A PRIORI ESTIMATES}

\subsection{PRELIMINARIES}

We note that the solution of (2. 15) has the full regularity, namely $u^{\epsilon, \nu} \in W^{2, p}(\Omega)$, in particular $u^{\epsilon, \nu} \in C^{1}(\bar{\Omega})$. We prove first

Lemma 3.1 We have the estimates

$$
\left|u^{\epsilon, \nu}(x)\right| \leq \frac{M^{\nu}}{\beta^{\nu}}
$$

PROOF:

Note first that, from (2.8),(2.10) one has

$$
\left.H^{\epsilon, \nu}(x, s, \xi)\right|_{\left\{\xi^{\nu}=0\right\}} \geq \beta^{\nu} s^{\nu}-M^{\nu}, \text { if } s^{\nu}>0
$$

and from (2.9),(2. 10)

$$
\left.H^{\epsilon, \nu}(x, s, \xi)\right|_{\left\{\xi^{\nu}=0\right\}} \leq \beta^{\nu} s^{\nu}+M^{\nu}, \text { if } s^{\nu}<0 .
$$

The function $u^{\epsilon, \nu}(x)$ being continuous in $\bar{\Omega}$ attains its maximum in $x^{\epsilon}$ (we omit to write the dependence in $\nu$ ). Suppose the maximum is strictly positive, then $x^{\epsilon} \in \Omega$. From the Maximum Principle, we have

$$
\left.H^{\epsilon, \nu}\left(x, u^{\epsilon}\left(x^{\epsilon}\right), D u^{\epsilon}\left(x^{\epsilon}\right)\right)\right|_{\left\{\xi^{\nu}=0\right\}} \leq 0
$$

so, using (3. 24), we deduce

$$
u^{\epsilon, \nu}\left(x^{\epsilon}\right) \leq \frac{M^{\nu}}{\beta^{\nu}}
$$

if $u^{\epsilon, \nu}\left(x^{\epsilon}\right)>0$, and this inequality is obvious if $u^{\epsilon, \nu}\left(x^{\epsilon}\right) \leq 0$. A similar inequality is proven for the minimum, using this time (3. 25). The result (3. 23) is thus obtained.

We shall now make use of the growth assumptions (2. 13),(2. 14). We first notice that we can write

$$
H^{\epsilon, \nu}(x, s, \xi)=Q^{\epsilon, \nu}(x, s, \xi) \cdot \xi^{\nu}+H_{0}^{\epsilon, \nu}(x, s, \xi)
$$

with the properties

$$
\begin{aligned}
& Q^{\epsilon, \nu}, \text { measurable, continuous in } s, \xi, \text { for } \xi^{\nu} \neq 0 \\
& \left|Q^{\epsilon, \nu}(x, s, \xi)\right| \leq K^{\nu}|\xi|, \nu=1, \cdots, N-1 \\
& Q^{\epsilon, N}=Q^{\epsilon, N-1}
\end{aligned}
$$


and

$$
\left|H_{0}^{\epsilon, \nu}(x, s, \xi)\right| \leq \sum_{\{\mu=1\}}^{\nu} K_{\mu}^{\nu}\left|\xi^{\mu}\right|^{2}+k^{\nu}(x), \nu=1, \cdots, N
$$

where the constants not yet defined are $K_{\mu}^{N}$ defined as follows

$$
K_{\mu}^{N}=K^{N}+\frac{1}{2} K^{N-1}, \mu=1, \cdots, N-1 ; K_{N}^{N}=K^{N}+K^{N-1} .
$$

Indeed, we set, for $\nu=1, \cdots, N-1$

$$
\sigma^{\epsilon, \nu}(x, s, \xi)=\frac{H^{\epsilon, \nu}(x, s, \xi)}{K^{\nu}|\xi|\left|\xi^{\nu}\right|+\sum_{\{\mu=1\}}^{\nu} K_{\mu}^{\nu}\left|\xi^{\mu}\right|^{2}+k^{\nu}(x)}
$$

and successively

$$
Q^{\epsilon, \nu}(x, s, \xi)=K^{\nu} \sigma^{\epsilon, \nu}(x, s, \xi)|\xi| \frac{\xi^{\nu}}{\left|\xi^{\nu}\right|}
$$

for $\nu=1, \cdots, N-1$, and $Q^{N}=Q^{N-1}$. Then we set

$$
H_{0}^{\epsilon, \nu}(x, s, \xi)=H^{\epsilon, \nu}(x, s, \xi)-Q^{\epsilon, \nu}(x, s, \xi) \cdot \xi^{\nu}
$$

then, it is easy to check that (3. 28), (3. 29), (3. 30), (3. 31) are verified.

We then proceed with a fundamental inequality. For simplicity at this stage, we shall omit to write explicitly $\epsilon$, since all estimates will be uniform with respect to $\epsilon$. We call

$$
\rho=\max _{\nu} \frac{M^{\nu}}{\beta^{\nu}}
$$

which is an $L^{\infty}$ bound for the solution of (2. 15), which we call temporarily $u^{\nu}$, without $\epsilon$. To any solution $u=\left(\cdots, u^{\nu}, \cdots\right)$, we associate a constant vector $c$, such that

$$
\|c\| \leq \rho
$$

and we write

$$
\tilde{u}=u-c .
$$

Let also

$$
\psi \geq 0, \psi \in H^{1} \cap L^{\infty}(\Omega),\left.\psi\right|_{\partial \Omega}=0 \text { if } c \neq 0 .
$$

We introduce the notation

$$
\beta(x)=\exp x-x-1
$$

and the map $X(s): R^{N} \rightarrow R^{N}$ defined backwards by the formulas

$$
\begin{aligned}
& X^{N}(s)=\exp \left[\beta\left(\gamma^{N} s^{N}\right)+\beta\left(-\gamma^{N} s^{N}\right)\right] \\
& X^{\nu}(s)=\exp \left[\beta\left(\gamma^{\nu} s^{\nu}\right)+\beta\left(-\gamma^{\nu} s^{\nu}\right)+X^{\nu+1}(s)\right], \nu=1, \cdots, N-1
\end{aligned}
$$

where $\gamma^{\nu}$ are positive constants ans $s=\left(s^{1}, \cdots, s^{N}\right)$. We note the formula

$$
\frac{\partial X^{\nu}}{\partial s^{\mu}}=\left\{\begin{array}{l}
0 \text { if } \mu<\nu \\
\gamma^{\mu} X^{\nu} \cdots X^{\mu}\left(\beta^{\prime}\left(\gamma^{\mu} s^{\mu}\right)-\beta^{\prime}\left(-\gamma^{\mu} s^{\mu}\right)\right) \text { if } \mu \geq \nu
\end{array}\right.
$$

We call

$$
X(x)=X(\tilde{u}(x))
$$


hence clearly

$$
D X^{\nu}=\sum_{\{\mu=\nu\}}^{N} \gamma^{\mu} X^{\nu} \cdots X^{\mu}\left(\beta^{\prime}\left(\gamma^{\mu} \tilde{u}^{\mu}\right)-\beta^{\prime}\left(-\gamma^{\mu} \tilde{u}^{\mu}\right)\right) D u^{\mu}
$$

from which we deduce the estimates

$$
\begin{aligned}
& |D X| \leq c(\rho)|\tilde{u}||D u| \\
& 0 \leq X(x)-X_{0} \leq c(\rho)|\tilde{u}|^{2}
\end{aligned}
$$

where in the sequel, $c(\rho)$ denotes a constant depending only of $\rho$ (this assumes that the constants $\gamma^{\mu}$ depend only of $\rho$ ), and $X_{0}$ is the value of $X(s)$ for $s=0$. We have

$$
X_{0} \geq 1
$$

We state the

Proposition 3.1 We assume (2. 1), (2. 2), (2. 3), (2. 13), (2. 14), a solution u of (2. 15), bounded by $\rho$. There exist constants $\gamma^{\nu}(\rho), c(\rho)$ such that, for any constant vector $c$ satisfying (3. 38), and any $\psi$ such that (3. 39) holds, one has

$$
\int_{\Omega} a_{i j} \frac{\partial X^{1}}{\partial x_{j}} \frac{\partial \psi}{\partial x_{i}} d x+\alpha \int_{\Omega} \psi|D u|^{2} d x \leq c(\rho) \int_{\Omega} \psi \sum_{\{\nu=1\}}^{N} k^{\nu} d x
$$

PROOF:

We take as a test function in $(2.15)$

$$
v^{\nu}=\psi \gamma^{\nu}\left(\beta^{\prime}\left(\gamma^{\nu} \tilde{u}^{\nu}\right)-\beta^{\prime}\left(-\gamma^{\nu} \tilde{u}^{\nu}\right)\right) \prod_{\mu=1}^{\nu} X^{\mu}
$$

then

$$
\begin{gathered}
\int_{\Omega}-\operatorname{div}\left(a(x) D u^{\nu}\right) v^{\nu} d x=\int_{\Omega} a_{i j} \frac{\partial u^{\nu}}{\partial x_{j}} \frac{\partial \psi}{\partial x_{i}} \gamma^{\nu}\left(\beta^{\prime}\left(\gamma^{\nu} \tilde{u}^{\nu}\right)-\beta^{\prime}\left(-\gamma^{\nu} \tilde{u}^{\nu}\right)\right) \prod_{\mu=1}^{\nu} X^{\mu} d x+ \\
+\int_{\Omega} a_{i j} \frac{\partial u^{\nu}}{\partial x_{j}} \psi \gamma^{\nu 2}\left(\beta^{\prime \prime}\left(\gamma^{\nu} \tilde{u}^{\nu}\right)+\beta^{\prime \prime}\left(-\gamma^{\nu} \tilde{u}^{\nu}\right)\right) \frac{\partial u^{\nu}}{\partial x_{i}} \prod_{\mu=1}^{\nu} X^{\mu} d x+ \\
+\int_{\Omega} a_{i j} \frac{\partial u^{\nu}}{\partial x_{j}} \psi \gamma^{\nu}\left(\beta^{\prime}\left(\gamma^{\nu} \tilde{u}^{\nu}\right)-\beta^{\prime}\left(-\gamma^{\nu} \tilde{u}^{\nu}\right)\right) \frac{\partial}{\partial x_{i}} \prod_{\mu=1}^{\nu} X^{\mu} d x \\
=I+I I+I I I .
\end{gathered}
$$

Then, one checks easily that

$$
\begin{gathered}
I=\int_{\Omega} a_{i j} \frac{\partial X^{1}}{\partial x_{j}} \frac{\partial \psi}{\partial x_{i}} d x \\
I I \geq \alpha \int_{\Omega} \psi\left|D u^{\nu}\right|^{2} \gamma^{\nu 2}\left(\exp \gamma^{\nu} \tilde{u}^{\nu}+\exp -\gamma^{\nu} \tilde{u}^{\nu}\right) \prod_{\mu=1}^{\nu} X^{\mu} d x \\
I I I=\int_{\Omega} a_{i j} \frac{\partial F^{\nu}}{\partial x_{j}} \frac{\partial F^{\nu}}{\partial x_{i}} \psi \prod_{\mu=1}^{\nu} X^{\mu} d x
\end{gathered}
$$

where

$$
F^{\nu}=\log X^{\nu}
$$


Next, we have

$$
\begin{gathered}
\sum_{\nu=1}^{N-1} \int_{\Omega} Q^{\nu} v^{\nu} d x=\sum_{\nu=1}^{N-1} \int_{\Omega} \psi Q^{\nu}\left(D F^{\nu}-D X^{\nu+1}\right) \prod_{\mu=1}^{\nu} X^{\mu} d x \\
=\sum_{\nu=1}^{N-1} \int_{\Omega} \psi\left(Q^{\nu}-Q^{\nu-1}\right) D F^{\nu} \prod_{\mu=1}^{\nu} X^{\mu} d x-\int_{\Omega} \psi Q^{N-1} D F^{N} \prod_{\mu=1}^{N} X^{\mu} d x
\end{gathered}
$$

where we have set $Q^{0}=0$. Since $Q^{N-1}=Q^{N}$, it follows that

$$
\sum_{\nu=1}^{N} \int_{\Omega} Q^{\nu} v^{\nu} d x=\sum_{\nu=1}^{N-1} \int_{\Omega} \psi \tilde{Q}^{\nu} D F^{\nu} \prod_{\mu=1}^{\nu} X^{\mu} d x
$$

where

$$
\tilde{Q}^{\nu}=Q^{\nu}-Q^{\nu-1}, \nu=1, \cdots, N-1 .
$$

Collecting results and performing additional majorations we obtain

$$
\begin{gathered}
\int_{\Omega} a_{i j} \frac{\partial X^{1}}{\partial x_{j}} \frac{\partial \psi}{\partial x_{i}} d x+ \\
+\alpha \sum_{\nu=1}^{N} \int_{\Omega} \psi\left|D u^{\nu}\right|^{2} \gamma^{\nu 2}\left(\exp \gamma^{\nu} \tilde{u}^{\nu}+\exp -\gamma^{\nu} \tilde{u}^{\nu}\right) \prod_{\mu=1}^{\nu} X^{\mu} d x \\
+\sum_{\nu=1}^{N} \int_{\Omega} \psi H_{0}^{\nu} \gamma^{\nu}\left(\exp \gamma^{\nu} \tilde{u}^{\nu}-\exp -\gamma^{\nu} \tilde{u}^{\nu}\right) \prod_{\mu=1}^{\nu} X^{\mu} d x \leq \frac{1}{4} \sum_{\nu=1}^{N-1} \int_{\Omega} \psi\left(\frac{a+a^{*}}{2}\right)^{-1} \tilde{Q}^{\nu} \cdot \tilde{Q}^{\nu} \prod_{\mu=1}^{\nu} X^{\mu} d x .
\end{gathered}
$$

Thanks to the properties (3. 30), (3. 31), it follows that

$$
\begin{gathered}
\int_{\Omega} a_{i j} \frac{\partial X^{1}}{\partial x_{j}} \frac{\partial \psi}{\partial x_{i}} d x+ \\
+\alpha \sum_{\nu=1}^{N} \int_{\Omega} \psi\left|D u^{\nu}\right|^{2} \gamma^{\nu 2}\left(\exp \gamma^{\nu} \tilde{u}^{\nu}+\exp -\gamma^{\nu} \tilde{u}^{\nu}\right) \prod_{\mu=1}^{\nu} X^{\mu} d x \leq \\
\sum_{\nu=1}^{N} \int_{\Omega} \psi\left|D u^{\nu}\right|^{2}\left[\frac{1}{4 \alpha} \sum_{\sigma=1}^{N-1}\left(K^{\sigma}+K^{\sigma-1}\right)^{2}\right. \\
\left.+\sum_{\sigma=\nu}^{N} \gamma^{\sigma} K_{\nu}^{\sigma}\left|\exp \gamma^{\sigma} \tilde{u}^{\sigma}-\exp -\gamma^{\sigma} \tilde{u}^{\sigma}\right|\right] \prod_{\mu=1}^{\sigma} X^{\mu} d x+ \\
+\sum_{\nu=1}^{N} \int_{\Omega} \psi \gamma^{\nu} k^{\nu}\left|\exp \gamma^{\nu} \tilde{u}^{\nu}-\exp -\gamma^{\nu} \tilde{u}^{\nu}\right| \prod_{\mu=1}^{\nu} X^{\mu} d x .
\end{gathered}
$$

Suppose the constants $\gamma^{\nu}(\rho)$ are chosen so that

$$
\begin{aligned}
& \alpha \gamma^{\nu 2}-2 \gamma^{\nu} K_{\nu}^{\nu} \geq \frac{1}{4 \alpha}\left[\sum_{\sigma=1}^{\nu}\left(K^{\sigma}+K^{\sigma-1}\right)^{2}+\right. \\
& \left.+\sum_{\sigma=\nu+1}^{N-1}\left(K^{\sigma}+K^{\sigma-1}\right)^{2} \prod_{\mu=\nu+1}^{\sigma} X^{\mu}\right]+ \\
& +\sum_{\sigma=\nu+1}^{N} \gamma^{\sigma} K_{\nu}^{\sigma}\left|\exp \gamma^{\sigma} \tilde{u}^{\sigma}-\exp -\gamma^{\sigma} \tilde{u}^{\sigma}\right| \prod_{\mu=\nu+1}^{\sigma} X^{\mu}
\end{aligned}
$$


and $c(\rho)$ is such that

$$
\gamma^{\nu}\left|\exp \gamma^{\nu} \tilde{u}^{\nu}-\exp -\gamma^{\nu} \tilde{u}^{\nu}\right| \prod_{\mu=1}^{\nu} X^{\mu} \leq c(\rho)
$$

then the result (3. 44) follows. The constants $\gamma^{\nu}(\rho)$ can be defined by the relations (3. 45) backwards, observing that $X^{\mu}$ can be majorized by a number depending only on $\rho, \gamma^{\mu}, \cdots, \gamma^{N}$.

The proof has been completed.

\subsection{ESTIMATES}

We begin by stating the following result concerning the $H_{0}^{1}$ estimates

Proposition 3.2 We have the estimate

$$
\alpha \int_{\Omega}\left|D u^{\epsilon}\right|^{2} d x \leq c(\rho)
$$

PROOF :

One just pick $c=0$ and $\psi=1$ in (3. 44). The result follows immediately.

We then proceed with the Hlder estimate, which is essential in the case of systems

Proposition 3.3 For $\delta<\delta_{0}=1-\frac{n}{2 q}$, one has the estimate

$$
\left|u^{\epsilon, \nu}\right|_{C^{\delta}} \leq c_{\delta}(\rho)
$$

We begin by introducing the Green function, with respect to a point $x_{0} \in \Omega$. Let $Q$ be a ball such that $\bar{\Omega} \subset Q$. The Green function is the solution $G=G^{x_{0}}$ of the equation

$$
\int_{Q} a D \phi \cdot D G d x=\phi\left(x_{0}\right), \forall \phi \in C_{0}^{\infty}(Q)
$$

Moreover, $G$ satisfies the estimates

$$
c_{0}\left|x-x_{0}\right|^{2-n} \leq G(x) \leq c_{1}\left|x-x_{0}\right|^{2-n}
$$

for all $x$ in a neighborhood of $x_{0}$, whose closure is contained in $Q$. In particular, (3. 50) holds for $x \in \bar{\Omega}$. The constants $c_{0}, c_{1}$ depend only on $\alpha, \alpha_{0}$, therefore they do not depend on $\epsilon$, whereas $G$ depends on $\epsilon$.

The next ingredient is the cut-off function. Let $\tau(x)$ be a smooth function such that $0 \leq \tau \leq 1$, and

$$
\tau(x)=1, \forall x \text { such that }|x| \leq 1, \tau(x)=1, \forall x \text { such that }|x| \geq 2 .
$$

We define

$$
\tau_{R}(x)=\tau\left(\frac{x-x_{0}}{R}\right)
$$

and we denote by $B_{R}=B_{R}\left(x_{0}\right)$ the ball of center $x_{0}$ and of radius $R$. We assume $R \leq R_{0}$. An essential element in the proof of Proposition 3.3 is the following

Lemma 3.2 We have the inequality

$$
\int_{B_{R}}\left|D u^{\epsilon}\right|^{2}\left|x-x_{0}\right|^{2-n} d x \leq C \int_{B_{4 R}-B_{R}}\left|D u^{\epsilon}\right|^{2}\left|x-x_{0}\right|^{2-n} d x+C R^{\beta}
$$

for all $R \leq R_{0}$ and $\beta \leq \beta_{0}=2-\frac{n}{q}$, with $C$ depending only on $\rho$. 
PROOF of LEMMA 3.2:

We apply (3. 44) with

$$
\psi=G \tau_{R}^{2}
$$

and

$$
c=c^{R}=\left\{\begin{array}{l}
0 \text { if } B_{2 R} \cap\left(R^{n}-\Omega\right) \neq \emptyset \\
\frac{1}{\left|B_{2 R}-B_{R}\right|} \int_{B_{2 R}-B_{R}} u d x \text { if } B_{2 R} \subset \Omega
\end{array}\right.
$$

We can also consider that $u$ is extended outside $\Omega$ with the value 0 .

We first notice that

$$
\alpha \int_{\Omega} G \tau_{R}^{2}|D u|^{2} d x \geq \alpha c_{0} \int_{B_{R}}|D u|^{2}\left|x-x_{0}\right|^{2-n} d x .
$$

Next

$$
\begin{gathered}
c(\rho) \int_{\Omega} G \tau_{R}^{2} \sum_{\{\nu=1\}}^{N} k^{\nu} d x \leq c(\rho)\left(\int_{B_{2 R}-B_{R}} G^{q^{\prime}} d x\right)^{\frac{1}{q^{\prime}}} \\
\leq c_{1} c(\rho)\left(\int_{B_{2 R}-B_{R}}\left|x-x_{0}\right|^{(2-n) q^{\prime}} d x\right)^{\frac{1}{q^{\prime}}}
\end{gathered}
$$

hence

$$
c(\rho) \int_{\Omega} G \tau_{R}^{2} \sum_{\{\nu=1\}}^{N} k^{\nu} d x \leq c(\rho) R^{2-\frac{n}{q}}
$$

where, of course the constant $c(\rho)$ is generic. We turn to the main term in (3. 44)

$$
\int_{\Omega} a_{i j} \frac{\partial X^{1}}{\partial x_{j}} \frac{\partial G \tau_{R}^{2}}{\partial x_{i}} d x=I+I I
$$

with

$$
I=2 \int_{\Omega} G \tau_{R} a_{i j} \frac{\partial X^{1}}{\partial x_{j}} \frac{\partial \tau_{R}}{\partial x_{i}} d x
$$

and

$$
I I=\int_{\Omega} \tau_{R}^{2} a_{i j} \frac{\partial X^{1}}{\partial x_{j}} \frac{\partial G}{\partial x_{i}} d x .
$$

Then, we can write

$$
I I=\int_{\Omega} a_{i j} \frac{\partial\left(\left(X^{1}-X_{0}^{1}\right) \tau_{R}^{2}\right)}{\partial x_{j}} \frac{\partial G}{\partial x_{i}} d x-2 \int_{\Omega} \tau_{R}\left(X^{1}-X_{0}^{1}\right) a_{i j} \frac{\partial \tau_{R}}{\partial x_{j}} \frac{\partial G}{\partial x_{i}} d x
$$

and from the definition of the Green function, see (3. 49)

$$
I I \geq-2 \int_{\Omega} \tau_{R}\left(X^{1}-X_{0}^{1}\right) a_{i j} \frac{\partial \tau_{R}}{\partial x_{j}} \frac{\partial G}{\partial x_{i}} d x .
$$

Making use of (3. 43), and performing easy majorations we obtain

$$
I+I I \geq-c(\rho)\left[\int_{\left(B_{2 R}-B_{R}\right) \cap \Omega} \frac{\left|u-c^{R}\right|^{2}}{R^{2}} G d x+\int_{B_{2 R}-B_{R}}|D u|^{2} G d x+I I I\right]
$$

where

$$
I I I=\int_{\left(B_{2 R}-B_{R}\right) \cap \Omega} G^{-1}|D G|^{2}\left|u-c^{R}\right|^{2} \tau_{R}^{2} d x .
$$

Note that

$$
\int_{\left(B_{2 R}-B_{R}\right) \cap \Omega} \frac{\left|u-c^{R}\right|^{2}}{R^{2}} G d x \leq c_{1} \int_{\left(B_{2 R}-B_{R}\right) \cap \Omega} \frac{\left|u-c^{R}\right|^{2}}{R^{2}}\left|x-x_{0}\right|^{2-n} d x
$$




$$
\leq c_{1} R^{2-n} \int_{\left(B_{2 R}-B_{R}\right) \cap \Omega} \frac{\left|u-c^{R}\right|^{2}}{R^{2}} d x
$$

and using Poincaré's inequality, we obtain

$$
\leq C R^{2-n} \int_{B_{2 R}-B_{R}}|D u|^{2} d x \leq C \int_{B_{2 R}-B_{R}}|D u|^{2}\left|x-x_{0}\right|^{2-n} d x .
$$

Therefor we have proven

$$
I+I I \geq-c(\rho)\left[\int_{B_{2 R}-B_{R}}|D u|^{2}\left|x-x_{0}\right|^{2-n} d x+I I I\right] .
$$

To estimate $I I I$, one introduces a new cut-off function, defined as follows

$$
\begin{aligned}
& \chi=0 \text { if }|x| \leq \frac{1}{2} \\
& \chi=\tau \text { if }|x| \geq 1
\end{aligned}
$$

and $\chi$ smooth, $0 \leq \chi \leq \tau$. We set

$$
\chi_{R}=\chi\left(\frac{x-x_{0}}{R}\right)
$$

and note that

$$
\chi_{R}=\tau_{R} \text {, outside } B_{R} \text {. }
$$

We take in (3. 49)

$$
\phi=G^{-\frac{1}{2}}\left|u-c^{R}\right|^{2} \chi_{R}^{2}
$$

noting that $\phi\left(x_{0}\right)=0$. We obtain the relation

$$
\frac{1}{2} \int a D G \cdot D G G^{-\frac{3}{2}}\left|u-c^{R}\right|^{2} \chi_{R}^{2} d x=\int a D\left(\left|u-c^{R}\right|^{2} \chi_{R}^{2}\right) \cdot D G G^{-\frac{1}{2}} d x
$$

Using now the system (2. 15), testing with $\left(u^{\nu}-c^{\nu, R}\right) G^{\frac{1}{2}} \chi_{R}^{2}$, it follows

$$
\begin{gathered}
\int a D u^{\nu} \cdot D u^{\nu} G^{\frac{1}{2}} \chi_{R}^{2} d x+\frac{1}{2} \int a D u^{\nu}\left(u^{\nu}-c^{\nu, R}\right) G^{-\frac{1}{2}} D G \chi_{R}^{2} d x+ \\
2 \int a D u^{\nu}\left(u^{\nu}-c^{\nu, R}\right) D \chi_{R} G^{\frac{1}{2}} d x+\int H^{\nu}\left(u^{\nu}-c^{\nu, R}\right) G^{\frac{1}{2}} \chi_{R}^{2} d x=0 .
\end{gathered}
$$

Hence

$$
\begin{gathered}
\int a D\left(\left|u-c^{R}\right|^{2} \chi_{R}^{2}\right) \cdot D G G^{-\frac{1}{2}} d x \leq 2 \int a D \chi_{R} \cdot D G G^{-\frac{1}{2}} \chi_{R}\left|u-c^{R}\right|^{2}- \\
-8 \int a D u^{\nu}\left(u^{\nu}-c^{\nu, R}\right) D \chi_{R} G^{\frac{1}{2}} d x+4 \int H^{\nu}\left(u^{\nu}-c^{\nu, R}\right) G^{\frac{1}{2}} \chi_{R}^{2} d x
\end{gathered}
$$

Using the quadratic growth of $H$, one checks easily that

$$
\begin{gathered}
\int a D\left(\left|u-c^{R}\right|^{2} \chi_{R}^{2}\right) \cdot D G G^{-\frac{1}{2}} d x \leq C \delta \int|D G|^{2} G^{-\frac{3}{2}}\left|u-c^{R}\right|^{2} \chi_{R}^{2} d x+ \\
+\frac{C}{\delta} \int_{\left(B_{2 R}-B_{\frac{R}{2}}\right) \cap \Omega} \frac{\left|u-c^{R}\right|^{2}}{R^{2}} G^{\frac{1}{2}} d x+ \\
+\int_{B_{2 R}-B_{\frac{R}{2}}}|D u|^{2} G^{\frac{1}{2}} d x+C R^{1-n+\frac{n}{q^{\prime}}}
\end{gathered}
$$


where $\delta$ is aribtrarily small. Combining with (3. 55)we obtain

$$
\begin{gathered}
\int|D G|^{2} G^{-\frac{3}{2}}\left|u-c^{R}\right|^{2} \chi_{R}^{2} d x \leq C \int_{B_{2 R}-B_{\frac{R}{2}}}|D u|^{2} G^{\frac{1}{2}} d x+ \\
+C \int_{\left(B_{2 R}-B_{\frac{R}{2}}\right) \cap \Omega} \frac{\left|u-c^{R}\right|^{2}}{R^{2}} G^{\frac{1}{2}} d x+C R^{1-n+\frac{n}{q^{\prime}}} .
\end{gathered}
$$

Finally, we can assert that

$$
I I I \leq C \int_{B_{2 R}-B_{\frac{R}{2}}}|D u|^{2}\left|x-x_{0}\right|^{2-n} d x+C R^{2-\frac{n}{q}} .
$$

Combining results, and changing $R$ by $2 R$, the result (3. 51) is obtained. This concludes the proof of Lemma 3.2.

\section{PROOF of PROPOSITION 3.3:}

Proceeding as for Lemma 3.2, with $\psi=G$ and $c=0$, one obtains

$$
\int|D u|^{2}\left|x-x_{0}\right|^{2-n} d x \leq C
$$

We can then use the hole filling technique of Widman (see K.O. WIDMAN [7]) to obtain

$$
\int_{B_{R}}|D u|^{2}\left|x-x_{0}\right|^{2-n} d x \leq C_{\beta} R^{\beta}, \beta<\beta_{0}=2-\frac{n}{q}
$$

and the result (3. 48) follows from the classical result of MORREY [4], with $\delta=\frac{\beta}{2}$.

\section{PROOF OF THEOREM 2.1}

\subsection{STRONG CONVERGENCE}

From PROPOSITIONS 3.2 and 3.3, we deduce that we can extract a subsequence such that

$$
u^{\epsilon, \nu} \rightarrow u^{\nu} \text { in } H_{0}^{1}(\Omega) \text { weakly and in } C^{0}(\bar{\Omega})
$$

Note also that $H^{\epsilon, \nu}\left(x, u^{\epsilon}, D u^{\epsilon}\right)$ remains bounded in $L^{1}(\Omega)$ and in $H^{-1}(\Omega)$ and thus we can assume that

$$
H^{\epsilon, \nu} \rightarrow \lambda^{\nu} \text { in } H^{-1}(\Omega) \text { weakly and in }\left(C^{0}(\bar{\Omega})\right)^{*} \text { weak star }
$$

Let us consider functions $\phi^{\nu}$ such that

$$
\begin{aligned}
& \phi^{\nu} \in C^{2}(\bar{\Omega}) \\
& \left.\phi^{\nu}\right|_{\partial \Omega}=0,\left.D \phi^{\nu}\right|_{\partial \Omega}=0
\end{aligned}
$$

and set

$$
\phi^{\epsilon, \nu}=\phi^{\nu}+D \phi^{\nu}\left(v^{\epsilon}-x\right) .
$$

Note that

$$
D \phi^{\epsilon, \nu}=\left(D v^{\epsilon}\right)^{*} D \phi^{\nu}+D^{2} \phi^{\nu}\left(v^{\epsilon}-x\right) .
$$


From the assumptions (2. 4),(2. 5),(2. 6), we then deduce

$$
\begin{aligned}
& \phi^{\epsilon, \nu} \rightarrow \phi^{\nu} \text { in } H_{0}^{1}(\Omega) \text { weakly } \\
& D \phi^{\epsilon, \nu}-\left(D v^{\epsilon}\right)^{*} D \phi^{\nu} \rightarrow 0 \text { in }\left(L^{2}(\Omega)\right)^{n} \text { weakly } \\
& a^{\epsilon} D \phi^{\epsilon, \nu} \rightarrow a D \phi^{\nu} \text { in }\left(L^{2}(\Omega)\right)^{n} \text { weakly } \\
& \operatorname{div} a^{\epsilon} D \phi^{\epsilon, \nu} \rightarrow \operatorname{div} a D \phi^{\nu} \text { in } H^{-1}(\Omega) \text { strongly }
\end{aligned}
$$

From (2. 4), (2. 7), we can assert that

$$
v^{\epsilon} \rightarrow x \text { in } C^{0}(\bar{\Omega})
$$

hence also

$$
\phi^{\epsilon, \nu} \rightarrow \phi^{\nu} \text { in } C^{0}(\bar{\Omega})
$$

We then state the Lemma

Lemma 4.1 We have the property

$$
\begin{aligned}
& \alpha \limsup \int \sum_{\nu}\left|D u^{\epsilon, \nu}-\left(D v^{\epsilon}\right)^{*} D \phi^{\nu}\right|^{2} d x \leq \\
& -\sum_{\nu} \int a D \phi^{\nu} \cdot D\left(u^{\nu}-\phi^{\nu}\right) d x-\sum_{\nu}<\lambda^{\nu}, u^{\nu}-\phi^{\nu}>
\end{aligned}
$$

PROOF:

Consider (2. 15), which we test with $u^{\epsilon, \nu}-\phi^{\epsilon, \nu}$. We deduce

$$
\begin{gathered}
\int a^{\epsilon} D\left(u^{\epsilon, \nu}-\phi^{\epsilon, \nu}\right) \cdot D\left(u^{\epsilon, \nu}-\phi^{\epsilon, \nu}\right) d x+ \\
-\int \operatorname{div}\left(a^{\epsilon} D \phi^{\epsilon, \nu}\right)\left(u^{\epsilon, \nu}-\phi^{\epsilon, \nu}\right) d x+\int H^{\epsilon, \nu}\left(u^{\epsilon, \nu}-\phi^{\epsilon, \nu}\right) d x=0 .
\end{gathered}
$$

Using (4. 61), (4.62) together with (4. 59), we obtain

$$
\begin{gathered}
\alpha \limsup \int \sum_{\nu}\left|D u^{\epsilon, \nu}-D \phi^{\epsilon, \nu}\right|^{2} d x \leq \\
\sum_{\nu} \int \operatorname{div}\left(a D \phi^{\nu}\right)\left(u^{\nu}-\phi^{\nu}\right) d x-\sum_{\nu}<\lambda^{\nu}, u^{\nu}-\phi^{\nu}>.
\end{gathered}
$$

Taking into account the second property (4. 61), we obtain (4. 63).

Now,we can assert that (4. 63) holds also for $\phi^{\nu} \in H_{0}^{1}(\Omega)$, since the second derivative has disappeared from the formula. Taking then $\phi^{\nu}=u^{\nu}$ we deduce

Proposition 4.1 We have the property

$$
D u^{\epsilon, \nu}-\left(D v^{\epsilon}\right)^{*} D u^{\nu} \rightarrow 0 \text { in }\left(L^{2}(\Omega)\right)^{n}
$$

\subsection{CONSTRUCTION OF THE LIMIT HAMILTONIAN}

Consider the sequence $H^{\epsilon, \nu}\left(x, s,\left(D v^{\epsilon}\right)^{*} \xi\right)$. From (2. 8) to ((2.12), we deduce

$$
\left|H^{\epsilon}\left(x, s,\left(D v^{\epsilon}\right)^{*} \xi\right)\right| \leq M+\varpi(|s|)\left(1+B^{2}|\xi|^{2}\right)+\gamma B|\xi|(1+B|\xi|)
$$

where

$$
M=\left(\sum_{\nu}\left(M^{\nu}\right)^{2}\right)^{\frac{1}{2}} .
$$


We follow the approach of BOCCARDO-MURAT [3]. Let $Z$ be a countable dense subset of $R^{N(n+1)}$, containing 0 and dense subsets of the subspaces $s^{\nu}=0$. There exists a subsequence such that

$$
H^{\epsilon, \nu}\left(x, s,\left(D v^{\epsilon}\right)^{*} \xi\right) \rightarrow H^{\nu}(x, s, \xi) \text { weakly in } L^{2}(\Omega), \forall s, \xi \in Z
$$

One checks easily that the properties (2. 8) to ((2.14) are satisfied for $H^{\nu}(x, s, \xi)$ on $Z$, with possibly different constants. These estimates, which imply a uniform continuity in $Z$ for bounded sets, permit to extend the definition of $H^{\nu}(x, s, \xi)$ to any pair $s^{\nu}, \xi^{\nu}$, and (2. 8) to ((2. 14) are satisfied. Moreover one has the property

$$
H^{\epsilon, \nu}\left(x, s,\left(D v^{\epsilon}\right)^{*} \xi\right) \rightarrow H^{\nu}(x, s, \xi) \text { weakly in } L^{2}(\Omega), \forall s, \xi
$$

We then asset the following result

Lemma 4.2 Let $\phi^{\nu} \in L^{\infty}(\Omega)$ and $\Gamma^{\nu} \in\left(L^{2}(\Omega)\right)^{n}$. Let $z^{\epsilon, \nu}$ be functions bounded in $L^{\infty}(\Omega)$, which converge in $L^{2}(\Omega)$ to functions $z^{\nu}$. Then one has the convergence property

$$
\int\left(H^{\epsilon, \nu}\left(x, \phi(x),\left(D v^{\epsilon}\right)^{*} \Gamma(x)\right)-H^{\nu}(x, \phi(x), \Gamma(x))\right) z^{\epsilon, \nu}(x) d x \rightarrow 0
$$

\section{PROOF:}

From (4. 67), one can assert that (4.68) holds whenever $\phi^{\nu}, \Gamma^{\nu}$ are bounded step functions. We can then consider approximations $\phi^{k, \nu}, \Gamma^{k, \nu}$ such that

$$
\begin{gathered}
\phi^{k, \nu} \rightarrow \phi^{\nu} \text { a.e. and }\left\|\phi^{k, \nu}\right\| \leq C \\
\Gamma^{k, \nu} \rightarrow \Gamma^{\nu} \text { in }\left(L^{2}(\Omega)\right)^{n} \text { and a.e. }
\end{gathered}
$$

$\phi^{k, \nu}, \Gamma^{k, \nu}$ being step functions. We write

$$
\begin{gathered}
\int\left(H^{\epsilon}\left(x, \phi(x),\left(D v^{\epsilon}\right)^{*} \Gamma(x)\right)-H(x, \phi(x), \Gamma(x))\right) z^{\epsilon, \nu}(x) d x \\
=\int\left(H^{\epsilon}\left(x, \phi(x),\left(D v^{\epsilon}\right)^{*} \Gamma(x)\right)-H^{\epsilon}\left(x, \phi^{k}(x), \Gamma^{k}(x)\right)\right) z^{\epsilon, \nu}(x) d x \\
+\int\left(H^{\epsilon}\left(x, \phi^{k}(x), \Gamma^{k}(x)\right)-H\left(x, \phi^{k}(x), \Gamma^{k}(x)\right)\right) z^{\epsilon, \nu}(x) d x \\
+\int\left(H\left(x, \phi^{k}(x), \Gamma^{k}(x)\right)-H(x, \phi(x), \Gamma(x))\right) z^{\epsilon, \nu}(x) d x \\
=I+I I+I I I .
\end{gathered}
$$

From uniform estimes, we can check that $I, I I I$ are bounded by $o(k)$ independent of $\epsilon$, and $o(k) \rightarrow 0$, as $k \rightarrow \infty$. Moreover, for fixed $k, I I \rightarrow 0$ as $\epsilon \rightarrow 0$. The result follows.

\subsection{END OF PROOF}

We can now complete the proof of Theorem 2.1. From (4. 64) and the uniform estimates (2. 11), (2. 12) it follows that

$$
H^{\epsilon, \nu}\left(x, u^{\epsilon}, D u^{\epsilon}\right)-H^{\epsilon, \nu}\left(x, u,\left(D v^{\epsilon}\right)^{*} D u\right) \rightarrow 0 \text { in } L^{1}(\Omega)
$$

Moreover from Lemma 4.2), we have

$$
H^{\epsilon, \nu}\left(x, u,\left(D v^{\epsilon}\right)^{*} D u\right)-H^{\nu}(x, u, D u) \rightarrow 0 \text { in } L^{1}(\Omega) \text { weakly }
$$


Therefore we deduce

$$
H^{\epsilon, \nu}\left(x, u^{\epsilon}, D u^{\epsilon}\right)-H^{\nu}(x, u, D u) \rightarrow 0 \text { in } L^{1}(\Omega) \text { weakly }
$$

Hence, we have

$$
\lambda^{\nu}=H^{\nu}(x, u, D u)
$$

From (4. 64, we have

$$
a^{\epsilon} D u^{\epsilon, \nu}-a^{\epsilon}\left(D v^{\epsilon}\right)^{*} D u^{\nu} \rightarrow 0 \text { in }\left(L^{2}(\Omega)\right)^{n}
$$

hence

$$
\operatorname{div} a^{\epsilon} D u^{\epsilon, \nu}-\operatorname{div}\left(a^{\epsilon}\left(D v^{\epsilon}\right)^{*} D u^{\nu}\right) \rightarrow 0 \text { in } H^{-1}(\Omega) \text { weakly }
$$

But, from the assumption (2.5) it follows

$$
\operatorname{div}\left(a^{\epsilon}\left(D v^{\epsilon}\right)^{*} D u^{\nu}\right) \rightarrow \operatorname{div}\left(a D u^{\nu}\right) \text { in } H^{-1}(\Omega) \text { weakly }
$$

hence finally
(4. 75$)$
$\operatorname{div} a^{\epsilon} D u^{\epsilon, \nu} \rightarrow \operatorname{div}\left(a D u^{\nu}\right)$ in $H^{-1}(\Omega)$ weakly

The proof has been completed.

\section{References}

[1] A. BENSOUSSAN, J. FREHSE, Regularity Results for Nonlinear Elliptic Systems and Applications, Springer-Verlag, Berlin, 2002.

[2] A. BENSOUSSAN, J. FREHSE, Smooth Solutions of Systems of Quasilinear Equations, in volume in memory of J.L. LIONS, dec 2001

[3] L. BOCCARDO, F. MURAT, Homogénéisation de problèmes quasi-linéaires, Atti del Convegno "Studio di problemi limite della analisi funzionale, Bressanone, (1982) , 13-51, Pitagora Editrice, Bologna

[4] C.B. MORREY Jr, Multiple Integrals in the Calculus of Variations, Springer-Verlag, Berlin, 1966.

[5] F. MURAT, L. TARTAR, Calcul des variations et homogénéisation, Eyrolles, coll DER-EDF, Lecture Notes, Paris

[6] L. TARTAR, Cours Peccot, Collège de France

[7] K. O. WIDMAN, Hlder continuity of solutions od elliptic equations, Manuscripta Math 5 (1971), 299- 308 